\title{
Why the Euler scheme in particle tracking is not enough: the shallow-sea pycnocline test case
}

\author{
Ulf Gräwe • Eric Deleersnijder • Syed Hyder Ali Muttaqi Shah • \\ Arnold Willem Heemink
}

Received: 16 September 2011 / Accepted: 3 January 2012 / Published online: 18 January 2012

(C) Springer-Verlag 2012

\begin{abstract}
During the last decades, the Euler scheme was the common "workhorse" in particle tracking, although it is the lowest-order approximation of the underlying stochastic differential equation. To convince the modelling community of the need for better methods, we have constructed a new test case that will show the shortcomings of the Euler scheme. We use an idealised shallow-water diffusivity profile that mimics the presence of a sharp pycnocline and thus a quasi-impermeable barrier to vertical diffusion. In this context, we study the transport of passive particles with or without negative buoyancy. A semi-analytic solutions is used to assess the performance of various numerical particle-tracking schemes (first- and secondorder accuracy), to treat the variations in the diffusivity
\end{abstract}

Responsible Editor: Dirk Olbers

Ulf Gräwe ( $\square)$

Leibniz Institute for Baltic Sea Research (IOW),

Seestraße 15, 18119 Warnemünde, Germany

e-mail: ulf.graewe@io-warnemuende.de

E. Deleersnijder

Institute of Mechanics, Materials and Civil Engineering

(IMMC), Université catholique de Louvain, 4 Avenue G.

Lemaître, 1348, Louvain-la-Neuve, Belgium

E. Deleersnijder

Earth and Life Institute (ELI), G. Lemaître Centre for

Earth and Climate Research (TECLIM), Université

catholique de Louvain, 2 Chemin du Cyclotron,

1348, Louvain-la-Neuve, Belgium

S.H.A.M. Shah · A.W. Heemink

Delft Institute of Applied Mathematics (DIAM),

Delft University of Technology, Mekelweg 4,

2628CD Delft, the Netherlands profile properly. We show that the commonly used Euler scheme exhibits a poor performance and that widely used particle-tracking codes shall be updated to either the Milstein scheme or second-order schemes. It is further seen that the order of convergence is not the only relevant factor, the absolute value of the error also is.

Keywords Particle tracking - Milstein scheme • Pycnocline $\cdot$ Diffusion $\cdot$ Residence time

\section{Introduction}

Over recent years, Lagrangian particle tracking became quite popular, as they offer a straightforward physical interpretation and a more intuitive understanding of the involved processes. As particle tracking deals with discrete elements and not with continuous fields like in the Eulerian framework (concentration fields), the equations of motion for both frameworks differ. However, the equivalence between both ways of describing the same process is given by the reinterpretation of a Fokker-Planck equation, i.e. a deterministic partial differential equation (PDE) (Arnold 1974). By contrast, particle tracking deals with stochastic differential equations (SDE) to describe the same process.

The application of particle tracking ranges from larvae dynamics (Christensen et al. 2007; Huret et al. 2007; Brochier et al. 2008), tracking of water masses (Blanke and Raynaud 1997; Soomere et al. 2011; Callies et al. 2011), oil spill modelling (Elliott et al. 1992; Mariano et al. 2011) or sediment dynamics (Lane and Prandle 2006; Krestenitis et al. 2007). Furthermore, particle tracking has been used to simulate dynamics in highly 
baroclinic environments (Blumberg et al. 2004; Proehl et al. 2005; North et al. 2006). Although the abovementioned applications are covering a wide range of topics, what they all have in common is that they are based on the Euler scheme (Maruyama 1955). This stochastic generalisation of the deterministic Euler scheme is the lowest-order numerical approximation for SDEs and is at best of first-order accuracy. This seems quite surprising, as recent developments in geophysical fluid dynamics propose the usage of high-order schemes for the advection or diffusion of momentum and tracer (Prather 1986; Pietrzak 1998; Iskandarani et al. 2005). Moreover, deterministic particle tracking, to quantify chaotic mixing in the ocean (D'Ovidio et al. 2004; Beron-Vera and Olascoaga 2009; Ohlmann and Mitarai 2010), is commonly based on fourth-order Runge-Kutta schemes. This discrepancy in usage of low-order stochastic particle-tracking schemes and high-order schemes in the deterministic case might be explained by two reasons. First, stochastic calculus deviates from the rather intuitive handling of integration, derivation or Taylor expansions as in the deterministic case. Thus, there is a lack of knowledge of stochastic calculus in the oceanic modelling community. Secondly, numerical approximations of SDEs are computationally expensive and, hence, are believed to limit their use. However, the marine modelling community is progressively realising that the Euler scheme is far from optimal (e.g. Stijnen et al. (2006); Spivakovskaya et al. (2007a); Gräwe and Wolff (2010)). Turning to higherorder schemes is one of the improvements that are now believed to be needed. Recently, Gräwe (2011) and Shah et al. (2011) discussed the performance of various stochastic numerical schemes and showed that not only the convergence of the error in a numerical approximation is important but also the total error. Furthermore, Gräwe (2011) showed that there exist schemes that are much more efficient (error vs. runtime) than the Euler scheme.

A common argument to excuse the usage of the Euler scheme is to vary the time step and to show that the solution converges (in a statistical sense) to a stable solution by reducing the time step. However, we will show that even the time step restrictions proposed by Visser (1997) does not guarantee that the numerical approximation converges to the true solution.

In recent years, a battery of test cases was developed to asses the accuracy and convergence of particletracking schemes (Thomson 1987; LaBolle et al. 2000; Brickman and Smith 2001; Deleersnijder et al. 2006a; Spivakovskaya et al. 2007a; Shah et al. 2011). Most of these test cases were designed to compare an analytical solution with the numerical approximations. Owing to the fact that analytical solutions are generally unavailable, only highly idealised test cases could be used. For example, the assumption that the vertical eddy diffusivity in shallow water has a parabolic profile is generally believed to be acceptable for an idealised test case. However, the assumption of a parabolic diffusivity implies that the water column is well mixed and no suppression of turbulence or vertical transport occurs, which is a well-observed feature in coastal oceanography. For instance, in shallow seas and estuaries, a pycnocline can act is a quasi-impermeable barrier to vertical diffusion. This variation in the vertical diffusivity is easily taken into account by Eulerian models, while obtaining a similar result in Lagrangian simulations is far from trivial. This was illustrated by Stijnen et al. (2006). Accordingly, it is necessary to develop Lagrangian test cases focussing on features that cannot be dealt with easily in a Lagrangian mode. In this paper, we construct a new benchmark that deals with an idealised pycnocline. This test case is used to assess the performance of different numerical particle-tracking schemes and to compare their efficiency. An additional positive side effect of the proposed test case is that the particletracking schemes are tested for the weak and strong convergence (Arnold 1974). Previous publications focused either on the weak convergence (Stijnen et al. 2006; Spivakovskaya et al. 2007a; Gräwe and Wolff 2010; Gräwe 2011) or on the strong convergence (Shah et al. 2011), but not on a combination of both.

The outline of the present paper is as follows: In Section 2, we briefly summarise the underlying theory for particle tracking. In Section 3, we discuss the construction of a test case that deals with an idealised pycnocline, which should act as a barrier to vertical diffusive transport. The numerical schemes, which shall be tested for accuracy, convergence and efficiency, are introduced in Section 4. In Section 5, we present the results and discuss the performance of the numerical schemes. In Section 5, we try to answer the question as to why the Euler scheme fails for the presented test case. Finally, the findings are summarised with a short conclusion.

\section{The Lagrangian model}

In the 1-D vertical Eulerian framework, the concentration of various constituents (suspended particular matter, pollutants, biological species etc.) obeys a PDE in the following form (neglecting source and sink terms):

$\partial_{t} C=\partial_{z}\left(w C+K(z) \partial_{z} C\right)$ 
Thus, Eq. 1 is an advection-diffusion equation for the concentration field $C(z, t)$ of a passive tracer that has a sinking velocity $w . K(z)$ represents the vertical eddy diffusivity.

Instead of solving the PDE, it is possible to transform the whole solution process into the solution of an SDE also called Langevin equation. The basic idea is to interpret the concentration field $C(z, t)$ as a transition density field and reinterpret Eq. 1 as a Fokker-Planck equation, i.e. a deterministic PDE with regard to transition density functions. This lead to the following SDE defined in the Îto sense (Arnold 1974):

$\mathrm{d} Z(t)=\left(w+\partial_{z} K(z)\right) d t+\sqrt{2 K(z)} d W(t)$

Here $Z(t)$ is the position vector of the particles and $d W(t)$ is a Wiener noise increment with the following properties. $W(t)$ is a Gaussian process with independent increments for which the following expressions hold

$$
\langle W(t)\rangle=0 ; \operatorname{Std}(W(t)-W(s))=\sqrt{|t-s|} .
$$

Therefore, the noise process has a vanishing mean $\langle\cdot\rangle$, its standard deviation scales as $\sqrt{d t}$ and the increments are uncorrelated. For convenience, Eq. 2 can be further simplified to

$$
\mathrm{d} Z(t)=a(z) \mathrm{d} t+b(z) \mathrm{d} W(t),
$$

where $a=w+\partial_{z} K(z)$ represents the deterministic part and $b=\sqrt{2 K(z)}$ is the stochastic part. Again Eqs. 4 and 2 are only valid in the Itto interpretation (Arnold 1974).

To evaluate the accuracy or order of convergence of a stochastic scheme, two cases have to be distinguished. For SDEs, the convergence is separated into weak and strong (Arnold 1974; Kloeden and Platen 1999). A method is said to have a weak/strong order of convergence of $\gamma$ if there exists a constant $\Lambda$ such that

$$
\begin{aligned}
\left|\left\langle p\left(Z_{n}\right)\right\rangle-\langle p(Z(\tau))\rangle\right| & \leq \Lambda \Delta t^{\gamma}: \text { weak } \\
\left\langle\left|Z_{n}-Z(\tau)\right|\right\rangle & \leq \Lambda \Delta t^{\gamma}: \text { strong },
\end{aligned}
$$

for any fixed $\tau=n \Delta t \in[0, T]$ and $\Delta t$ sufficiently small. $Z_{n}$ represents the true solution and $Z(\tau)$ is the approximation. The function $p(\cdot)$ is an arbitrary function (not necessarily a probability density function) with polynomial growth. This implies that all the moments of the distribution (and hence the distribution itself) are approximated with the desired accuracy. The weak criterion asks for the difference in a distribution, whereas the strong criterion accounts for the difference in the trajectory.

\section{Construction of the test case}

\subsection{Motivation}

The starting point is the test case of Stijnen et al. (2006). The authors performed Lagrangian simulations in a shallow coastal region (the coastal zone of the Netherlands). The challenge they were faced with was the vertical stratification due to salinity contrasts, caused by river run-off. The stratification, which is associated with a rather thin pycnocline, is a quasi-impermeable barrier to vertical diffusive or turbulent transport. Stijnen et al. (2006) showed that this is easily taken into account by Eulerian models, while obtaining a similar result in Lagrangian simulations is far from trivial. They could show that the pycnocline was no significant barrier to diffusion when the Lagrangian Euler scheme was used. However, when using a higher-order particle-tracking scheme, the pycnocline remained almost impermeable to diffusive fluxes-as it is supposed to be. Unfortunately, Stijnen et al. (2006) used a fairly large time step and did not do a detailed analysis of the reason why the Euler scheme fails. As we believe that it is important to investigate in detail the advantages and disadvantages of tools we are using, we will tackle the questions they left open.

As a test case should run on a standard desktop computer, it is desirable that the CPU cost of each run is modest so that many runs can be performed within a reasonably small amount of time. This allows numerous schemes to be tested and an assessment of a wide spectrum of parameter values to be considered. This is why it is suggested to turn to a vertical problem in which transport is due to vertical turbulent diffusion, with the possible addition of settling processes (e.g. Spivakovskaya et al. (2007a); Gräwe and Wolff (2010); de Brauwere and Deleersnijder (2010)). Hence, horizontal transport processes are not taken into account. A pycnocline associated with a strong density gradient must be present so that the eddy diffusivity at the location of the pycnocline is negligible, thereby representing an impermeable barrier to vertical diffusive transport. In Fig. 1, a measured diffusivity profile in the presence of a sharp pycnocline is shown (van der Lee and Umlauf 2011). The large variation in the diffusivity is caused by a gradient in the salinity with a difference of $10 \mathrm{~g} / \mathrm{kg}$ over $20 \mathrm{~m}$. An important fact to realise is that the diffusivity can vary by several orders of magnitude over a short distance.

To construct a possible test case, we use the diffusivity profile in Fig. 1 as a blue print. We assume without any loss of generality that the pycnocline is located in the middle of the water column. Accordingly, 


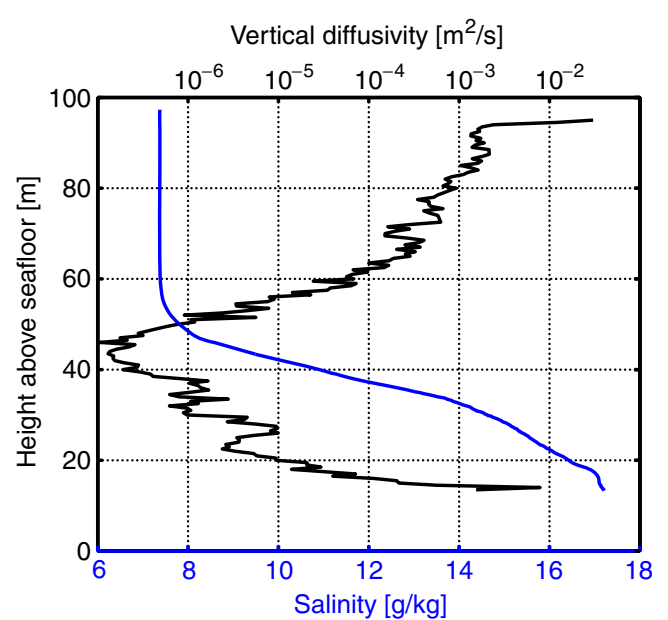

Fig. 1 Salinity and diffusivity profile measured in the Baltic Sea. For details of the measurement, see van der Lee and Umlauf (2011). Note that the upper $5 \mathrm{~m}$ and lower $10 \mathrm{~m}$ are not plotted

it is suggested that the idealised vertical eddy diffusivity be equal to:

$K(z)=\bar{K} \frac{2(1+a)(1+2 a)}{a^{2} H^{1+1 / a}}\left\{\begin{array}{c}z(H-2 z)^{1 / a} \\ 0 \leq z<H / 2 \\ (H-z)(2 z-1)^{1 / a} \\ 0 \leq z<H / 2\end{array}\right.$,

where $a$ is a constant that is larger than or equal to unity, $z$ is the distance to the seabed, which is located at $z=0$, while the sea surface is at $z=H$.

$\bar{K}$ is a free parameter, representing the depthaveraged diffusivity,

$\bar{K}=\frac{1}{H} \int_{0}^{H} K(z) \mathrm{d} z$.

The important tuning parameter is $a$, which controls the sharpness of the pycnocline. The dependence of the sharpness of the pycnocline on $a$ is depicted in Fig. 2. The eddy diffusivity is a linear function of the distance to the upper and lower boundaries of the domain: Such behaviour is generally regarded as acceptable for idealised test cases. In addition, the eddy diffusivity is zero at the pycnocline and in the vicinity of the latter. The parameter $a$ controls the steepness of the diffusivity profile. The larger the value of $a$, the larger the vertical diffusivity gradient in the vicinity of the pycnocline. For values of $a$ greater than 10 , one can generate pathologically thin interfaces which we will not consider. Note that setting $a=1$ will produce a double parabolic diffusivity profile.

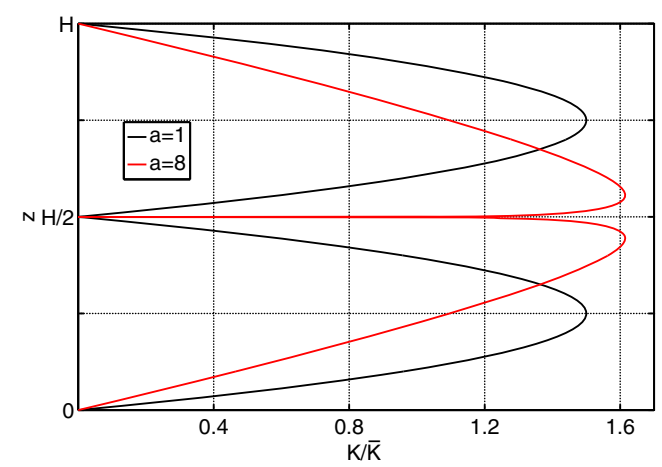

Fig. 2 Diffusivity profile $K$ for two different values of $a$

\subsection{Diffusive pycnocline crossing}

For the first test case, we will consider a pure diffusion problem; thus, we set in Eq. 1 the sinking velocity $w$ to zero. Moreover, for the sake of generality, it is convenient to reformulate the problem above using dimensionless variables. The latter are defined to be:

$t^{\prime}=\frac{t \bar{K}}{H^{2}} ; z^{\prime}=\frac{z}{H} ; K^{\prime}=\frac{K}{\bar{K}} ;$

From here on, only dimensionless quantities will be used; we will thus drop the primes. The domain of interest and the problem to be solved may be rewritten as follows:

$$
\begin{aligned}
& 0 \leq t ; 0 \leq z \leq 1 \\
& \partial_{t} C=\partial_{z}\left(K(z) \partial_{z} C\right) \\
& \left.K(z) \partial_{z} C\right|_{z=(0,1)}=0 \\
& C(0, z)=\delta\left(z-z_{0}\right) .
\end{aligned}
$$

The boundaries are treated as "no flux" boundaries, and the initial release is a Dirac function. For an analytical solution of the posed problem in the special case of $a=1$, the interested reader is referred to Spivakovskaya et al. (2007a).

However, the intention of the test case is not to reproduce the analytical solution. Our aim is to assess the ability of different numerical schemes to treat the pycnocline as the requested impermeable barrier. Thus, if we release particles in the upper half of the water column, a "perfect" scheme must exhibit zero concentration in the lower half of the water column at any time. This is easily achieved in Eulerian numerical models. In Lagrangian simulations, however, it is almost impossible to prevent some particles from crossing the pycnocline, thereby causing simulation errors that have to be assessed. To quantify to what extent the pycnocline is actually a barrier to vertical diffusion, $N$ particles may be released somewhere in the upper half of the water column. Since the number of particles 
has to remain constant, one could display the number of particles present in the lower half of the domain. As the whole modelling system outputs concentration fields (a detailed description is given by Gräwe (2011)), rather than individual trajectories, we use as error measure the following definition:

$\varepsilon=2 \int_{0}^{0.5} C(T, z) \mathrm{d} z$

with

$\int_{0}^{1} C(T, z) \mathrm{d} z=1$

thus, we integrate (sum) the particle concentration $C(T, z)$ at an arbitrary time $T$, in the lower half of the water column. If $\varepsilon=0$, no particles have crossed the pycnocline; if $\varepsilon=1$, the particles are uniformly distributed in the whole water column. Clearly, the lower $\varepsilon$, the better the scheme under considerations. Although we have a well-defined error measure, it must be realised that it is a non-linear one. At first, it can only vary between zero and one, and secondly if a scheme has an $\varepsilon$ of zero, it will remain zero by further reduction of the time step. Thus, there is obviously no further improvement.

To finish the introduction of the error measure, we have to define the integration time $T$, at which the evaluation of $\varepsilon$ is done. If $T$ is too small, the particle cloud has not reached the pycnocline, and if $T$ is large, the distribution is uniform over the whole water column, regardless of the used scheme. The vertical mixing time scale $T_{M}$ (with physical units) is of the order of:

$T_{M}=\frac{(H / 2)^{2}}{\bar{K}}=\frac{H^{2}}{4 \bar{K}}$.

$H / 2$ is related to the fact that the pycnocline is located in the middle of the water column. Therefore, $T_{M}$ represents a lower limit of the runtime. The dimensionless mixing time scale has a value of $T_{M}=1 / 4$. To deduce an upper bound for the $T$ is only possible by educated guessing. We have chosen to use the fourfold of $T_{M}$ as integration time. This is a trade-off between computational demand and accuracy.

An important point to mention is that although we are looking for the time evolution of a particle distribution and thus a weak convergence (Eq. 5), the crossing of the pycnocline tests for the strong convergence. This is related to the fact that the individual particle path in the vicinity of the pycnocline is important and therefore a strong error measure.

\subsection{Residence time}

In the previous test, the settling velocity $w$ was set to zero. This might be appropriate for tracking water parcels, but for buoyant particles, this assumption is no longer valid. However, imposing a finite sinking velocity makes it impossible to formulate an analytical solution. This even holds for $a=1$ and thus recovering the parabolic diffusivity profile. Nevertheless, an exact solution for the adjoint problem of finding the residence time $\theta(z)$ is known (Deleersnijder et al. 2006a, b). To obtain the residence time $\theta\left(z_{0}\right)$, a number of particles are released at a distance $z_{0}$ from the bottom, and the residence time is defined to be the mean time taken by the particles to settle in the sea bed.

To simplify the calculations in the following, it is again convenient to introduce dimensionless variables:

$t^{\prime}=\frac{t}{H / w} ;\left(z^{\prime}, z_{0}^{\prime}\right)=\frac{\left(z, z_{0}\right)}{H} ; K^{\prime}=\frac{K}{\bar{K}} ;$

Finally, the Peclet number $P e$ is defined to be

$P e=\frac{w H}{\bar{K}}$

and because only dimensionless variables will be used, we will drop again all primes from now on. Accordingly, the domain of interest is now defined as $0 \leq z \leq 1$. The initial and boundary conditions are defined as:

IC: $C(0, z)=\delta\left(z-z_{0}\right) \quad ; \quad \mathrm{BC}: \quad\left[C+K(z) \partial_{z} C\right]_{z=1}=0$.

The boundary at $z=0$ (seafloor) is a barrier to turbulent diffusion, and therefore, the diffusion flux must be zero at the bottom:

$\mathrm{BC}:\left.\quad K(z) \partial_{z} C\right|_{z=0}=0$.

In the case of a double parabolic diffusivity profile ( $a=$ 1 in Eq. 6), a closed-form expression of the residence times for the lower or upper part of the water column can be derived (Deleersnijder et al. 2006b). However, for the general case of $a \neq 1$, no closed-form expression exists, and one only has the following general formula (Deleersnijder et al. 2006a, b):

$\theta(z)=z+\int_{z}^{1} \exp \left[-P e \int_{z}^{\xi} \frac{\mathrm{d} \zeta}{K(\zeta)} \mathrm{d} \xi\right]$.

This integral can be evaluated numerically to any desired accuracy and thus be used as reference solution. The residence time profiles for $a=1$ and $a=5$ are 


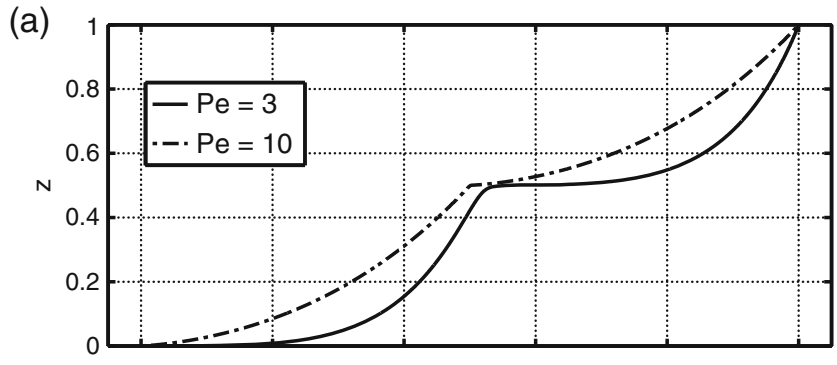

(b)

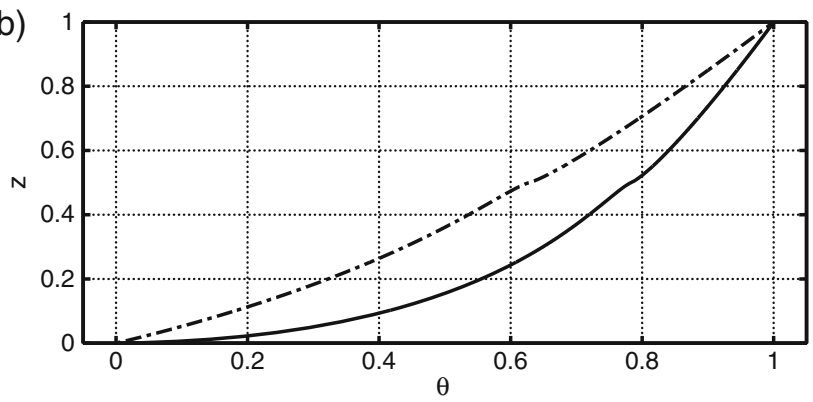

Fig. 3 Residence time $\theta$ for two different Peclet numbers $P e$, $\mathbf{a}$ for $a=1$ and $\mathbf{b} a=5$. These curves have been obtained from Eq. 9

shown in Fig. 3 for two different Peclet numbers. It can be seen that for higher values of $a$, the presence of the pycnocline at $z=0.5$ is not visible any longer. As the diffusivity vanishes at $z=0.5$, the particle behaviour in the vicinity of the pycnocline is controlled by the sinking velocity. If the value of the parameter $a$ increases, the thickness of the region (straddling the pycnocline) in which the diffusivity is small decreases. Accordingly, the impact of this region on the residence time obtained from Eq. 9 diminishes, which is why the influence of the pycnocline neighbourhood is less visible in the lower panel of Fig. $3(a=5)$ than in the upper one $(a=1)$.

To quantify the performance of the numerical algorithms, the root mean square error $\varepsilon$ is computed,

$\varepsilon=\sqrt{\frac{1}{N_{z}} \sum_{i=1}^{N_{z}}\left(\theta_{A}\left(z_{i}\right)-\theta_{S}\left(z_{i}\right)\right)^{2}}$

with $\theta_{A}\left(z_{i}\right)$ is the analytical solution (Eq. 9) and $\theta_{S}\left(z_{i}\right)$ is the prediction of the Lagrangian model. The analytical solution and the model prediction are computed at $N_{z}$ equidistant bins of the water column. In the previous section, we saw that the crossing of the pycnocline tests for the strong convergence. This is not the case for the residence time, as this is an integral quantity and therefore only a weak convergence benchmark.

\subsection{Time step restrictions}

To choose a proper time step in particle tracking is much more difficult than for instance in Eulerian ocean models. In the latter case, an upper limit is the ratio of the propagation speed of surface gravity waves (internal waves) and the grid spacing. As such a strict criterion does not exist for SDEs, the time step has to be selected either by sensitivity studies or from some general guidelines.

Penland (2003) proposed as a rule of thumb to estimate the smallest time scale of the system and divide this time scale by $2^{7}$. In our case, the smallest time scale is the mixing time scale $T_{M}$ (Eq. 8), such that we have a time step $\Delta t=(1 / 4) / 2^{7}=0.002$. However, this suggestion can only act as a first guess, since it does not take into account the sharpness of the pycnocline. Furthermore, one has to know in advance the smallest time scale of the system, which may be difficult to estimate.

To arrive at a better guess of the required time step, we will discuss the suggestions of Visser (1997). To choose a time step that resolves the variations in the diffusivity, Visser (1997) proposed a restriction on the time step for the integration:

$\Delta t \ll \min \left(\frac{1}{\partial_{z z} K}\right)$.

It is widely accepted that this formulation implies that $\Delta t$ must be at least one order of magnitude smaller than the reference time scale. However, the diffusivity profile (Eq. 6) has, as it is constructed, an inherent discontinuity in the curvature of the diffusivity at $z=$ 0.5 , which might cause problems. However, one has to note that the discontinuity only occurs in the derivative and curvature and not in the diffusivity profile itself. We do not have a jump in the diffusivity as used by LaBolle et al. (2000) or Spivakovskaya et al. (2007b). At $z=0.5$, the curvature of the diffusivity behaves as:

$\left.\partial_{z z} K(z)\right|_{z=0.5} \propto \lim _{z \rightarrow 0.5}(1-2 z)^{\frac{1-2 a}{a}}$,

Table 1 Time step limits based on Eq. 11 for different values of $a$ for the discretised version of the diffusivity profile (Eq. 6)

\begin{tabular}{lllll}
\hline$a$ & 1 & 2 & 4 & 8 \\
\hline$\Delta t_{\max }$ & $1 \times 10^{-3}$ & $4 \times 10^{-4}$ & $2 \times 10^{-4}$ & $1 \times 10^{-4}$ \\
\hline
\end{tabular}


Table 2 Settings for the diffusive pycnocline crossing and residence time test, with $N_{z}$ the number of vertical grid points and $N$ the total number of tracked particles

\begin{tabular}{llllll}
\hline & $\Delta t$ & $N_{z}$ & $N$ & $a$ & $P e$ \\
\hline Pycnocline crossing & $10^{-4}-10^{-6}$ & 100 & $10^{5}$ & 2 & 0 \\
a & $10^{-5}$ & 100 & $10^{5}$ & $1-10$ & 0 \\
b & & & & \\
Residence time & $10^{-4}-10^{-6}$ & 100 & $10^{5}$ & 2 & 3,12 \\
a & $10^{-5}$ & 100 & $10^{5}$ & $1-10$ & 3,12 \\
b &
\end{tabular}

hence, the curvature grows unbounded and the time step limit tends to zero. Nevertheless, as we approximate the diffusivity profile on a finite grid, the chosen time step depends strongly on the grid resolution. In Table 1 , we give an upper limit of the proposed time step for a vertical resolution of 100 equidistant grid points.

\subsection{Experimental settings}

Since the numerical schemes are tested for a wide range of parameters, in Table 2, the experimental settings are summarised. The number of vertical levels $N_{z}$ is limited to 100 , as this is similar to present day high-resolution ocean model discretisation. Using a higher/lower number or levels would only change the time step (Eq. 11), as the diffusivity profile is given analytically. To get a proper scaling of the error $\varepsilon$, the time step is varied by two orders of magnitude.

To estimate the concentration profiles, we resort to a simple box-counting approach, as using kernel estimates (Silverman 1986; Spivakovskaya et al. 2007a) would smooth the concentration profile at the pycnocline. The random numbers are generated by the "keep it simple stupid" (KISS) generator of Marsaglia (2003) in combination with the Ziggurat algorithm of Marsaglia and Tsang (2000) to map uniform random numbers to Gaussian ones.

\section{Numerical approximations}

In the following section, a short presentation of the used schemes is provided. A detailed description and discussion of the implementation may be found in Gräwe (2011).

\subsection{The Euler scheme (E1)}

A straightforward translation of Eq. 4 into a numerical scheme simply consists in replacing $\mathrm{d} t$ by a finite time step $\Delta t$ and $\mathrm{d} W$ by a discrete increment $\Delta W$. Therefore, the lowest-order approximation reads as:

$$
Z_{n+1}=Z_{n}+a \Delta t+b \Delta W_{n} .
$$

This is also known as Euler scheme (Maruyama 1955). As discussed after Eq. 3, the increment $\Delta W$ scales as $\sqrt{\Delta t}$; hence, the whole Euler scheme is only of order $\sqrt{\Delta t}$ in the sense of strong convergence and of order $\Delta t$ in the sense of weak convergence. The noise increment is drawn from a Gaussian distribution (Eq. 3). To compute the velocity and the diffusivity at the particle position, they need to be interpolated from the discrete grid. This is done via linear interpolation

\subsection{The Milstein scheme (M1)}

To develop numerical schemes that have a higher accuracy than the Euler scheme, one has to use the appropriate Taylor approximation of Eq. 4 (see, e.g. Arnold (1974) or Kloeden and Platen (1999)). The next higherorder approximation reads

$Z_{n+1}=Z_{n}+a \Delta t+b \Delta W_{n}+\frac{1}{2} b b^{\prime}\left(\Delta W_{n}^{2}-\Delta t\right)$,

where $b^{\prime}$ is the spatial derivative. This is also known as the Milstein scheme (Milstein 1974). Additional accuracy is gained by including information of the derivative of the noise term $b$. The noise increment $\Delta W$ is again drawn from a Gaussian distribution (Eq. 3). The interpolation is done linearly.

Although the Milstein scheme and Euler scheme have the same order of accuracy in terms of the weak convergence, they differ in the strong convergence. As we will later show, this is a crucial difference.

\subsubsection{The 1.5-order strong Taylor scheme (S15)}

By including further terms of the stochastic Taylor expansion, the next higher-order scheme is of 1.5-order accuracy in the strong convergence and second order in 
the weak sense. The 1.5-order strong Taylor scheme is given as:

$$
\begin{aligned}
Z_{n+1}= & Z_{n}+a \Delta t+b \Delta W_{n}+\frac{1}{2} b b^{\prime}\left(\Delta W_{n}^{2}-\Delta t\right) \\
& +a^{\prime} b \Delta Z_{n}+\frac{1}{2}\left(a a^{\prime}+\frac{1}{2} b^{2} a^{\prime \prime}\right) \Delta t^{2} \\
& +\left(a b^{\prime}+\frac{1}{2} b^{2} b^{\prime \prime}\right)\left(\Delta W_{n} \Delta t+\Delta Z_{n}\right) \\
& +\frac{1}{2} b\left(b b^{\prime \prime}+\left(b^{\prime}\right)^{2}\right)\left(\frac{1}{3} \Delta W_{n}^{2}-\Delta t\right) \Delta W_{n}(15)
\end{aligned}
$$

Additional to the noise increment $\Delta W$, a second random variable $\Delta Z$ is needed. $\Delta Z$ is also a Gaussian random variable (Eq. 3) with the following properties: $\left\langle\Delta Z_{n}\right\rangle=0$, variance $\left\langle\Delta Z^{2}\right\rangle=\frac{1}{3} \Delta t$ and covariance $\langle\Delta Z \Delta W\rangle=\frac{1}{2} \Delta t^{2}$. As terms like $a^{\prime \prime}, b^{\prime \prime}$ are needed, linear interpolation is not sufficient any longer. Thus, the interpolation of the diffusivity to the particle position is done via cubic spline interpolation (Lalescu et al. 2010).

\subsection{The second-order Milstein scheme (M2)}

This scheme is a simplification of the previous one. Therefore, only a weak approximation is needed, and some terms of Eq. 15 can be skipped. In contrast to Eq. 15, there is no need for a second random variable. The scheme was proposed by Milstein (1979) and is of second-order accuracy:

$$
\begin{aligned}
Z_{n+1}= & Z_{n}+a \Delta t+b \Delta W_{n}+\frac{1}{2} b b^{\prime}\left(\Delta W_{n}^{2}-\Delta t\right) \\
& +\frac{1}{2}\left((a b)^{\prime}+\frac{1}{2} b^{\prime \prime} b^{2}\right) \Delta W_{n} \Delta t+\frac{1}{2}\left(a a^{\prime}+\frac{1}{2} a^{\prime \prime} b^{2}\right) \Delta t^{2} .
\end{aligned}
$$

As above, the interpolation is done via cubic splines.

\subsubsection{The Heun scheme (PC1)}

The lowest-order predictor-corrector (PC) scheme reads as follows:

$Z_{n+1}=Z_{n}+\frac{1}{2}(a(\tilde{Z})+a) \Delta t+b \Delta W_{n}$

with

$\tilde{Z}=Z_{n}+a \Delta t+b \Delta W_{n}$.

Equation 17 is a stochastic version of the trapezoidal method also known as Heun scheme. Note that the predictor step Eq. 18 is only applied to the deterministic part, the stochastic part is not corrected to keep the numerical approximation consistent with Eq. 4 (Kloeden and Platen 1999). The Heun scheme is of first-order accuracy in the weak convergence and is therefore similar to the Euler scheme.

\subsubsection{The Platen two-step scheme (PC2)}

Another predictor-corrector scheme, which is of second order accuracy, was proposed by Kloeden and Platen (1999)

$$
Z_{n+1}=Z_{n}+\frac{1}{2}(a(\hat{Z})+a) \Delta t+\Phi
$$

with

$$
\begin{aligned}
\Phi=b \Delta W_{n} & +\frac{1}{2} b b^{\prime}\left(\Delta W_{n}^{2}-\Delta t\right) \\
& +\frac{1}{2}\left(a b^{\prime}+\frac{1}{2} b^{\prime \prime} b^{2}\right) \Delta W_{n} \Delta t
\end{aligned}
$$

and with the predictor

$$
\begin{aligned}
\hat{Z}=Z_{n} & +a \Delta t+\Phi+\frac{1}{2} a^{\prime} b \Delta W_{n} \Delta t \\
& +\frac{1}{2}\left(a a^{\prime}+\frac{1}{2} a^{\prime \prime} b^{2}\right) \Delta t^{2},
\end{aligned}
$$

where $\Delta W$ is again a Gaussian variable (Eq. 3). The interpolation is done via cubic splines.

Table 3 Summary of the different numerical schemes and related abbreviations. Shown are the theoretical order of convergence and the interpolation method used. Note that the strong order of convergence is not defined for all schemes

\begin{tabular}{lllll}
\hline Scheme & Short name & Weak order & Strong order & Interpolation \\
\hline Euler & E1 & 1 & 0.5 & Linear \\
Milstein 1st order & M1 & 1 & 1 & Linear \\
Strong Taylor & S15 & 2 & 1.5 & Cubic splines \\
Milstein 2nd order & M2 & 2 & - & Cubic splines \\
Heun & PC1 & 1 & 0.5 & Linear \\
Platen two-step & PC2 & 2 & - & Cubic splines \\
\hline
\end{tabular}




\subsection{Summary of used schemes}

Table 3 summarises the numerical schemes, the theoretical order of convergence, and the applied interpolation method and introduces the short notations used.

\section{Results and discussion}

\subsection{The pycnocline crossing test case}

In Fig. 4, the time evolution of a point release of particles at $z=0.75$ is shown. One can clearly see that for the $E 1$ scheme, the pycnocline is not a true barrier to diffusion. This even holds for small values of $a$. The M2 scheme shows for $a=1$ no crossing of particles of the pycnocline. For $a=4$ (Fig. 4 d), there is a leakage of particles into the lower half of the water column. By simple visual inspection, it is obvious that the results obtained with the Euler scheme are completely wrong. Furthermore, variations of the time step would not reveal this failure as we are already using a time step which is $1 / 100$ of the time step suggested by Visser (1997).

\subsubsection{Variation of the time step $\Delta t$}

To have a more quantitative performance measure, in Fig. 5, the scaling of the error $\varepsilon$ (Eq. 7) for different time steps is presented. Figure 5 a indicates that the $E 1$ and $P C 1$ scheme do not treat the pycnocline as a barrier by varying the time step over two orders of magnitude. Both schemes show only a slow convergence to the true solution. The other schemes show a proper performance with a convergence of the error by decreasing the time step. Figure 5 a further shows that for time steps smaller than $10^{-5}$, the $M 1, M 2, P C 2$ and
S15 schemes have a vanishing error. This suggests that the time step should be a factor of 100 smaller than the limit proposed by Visser (1997) (Eq. 11).

It is not surprising to see that the $S 15$ scheme shows the best performance. As the diffusive crossing of the pycnocline is a test that seeks for a strong error convergence, the $S 15$ scheme can outperform the other schemes. This is because the $S 15$ scheme is by construction a strong scheme and exhibits the highest theoretical accuracy within all schemes considered here.

Increasing the pycnocline sharpness by setting $a=$ 3 leads to an overall degradation of the performance for all schemes (Fig. $5 \mathrm{~b}$ ). Only for a time step of $\Delta t=$ $10^{-6}$ the pycnocline remains a impermeable boundary (except for the $E 1$ and $P C 1$ scheme).

The error convergence in Fig. 5 is plotted on semilogarithmic axes. As the error decreases nearly linearly for all schemes, this implies an exponential error convergence. It is further visible that the $E 1$ and $P C 1$ scheme exhibit a much slower "error decay" timescale than the four other schemes.

\subsubsection{Variation of the pycnocline sharpness}

To have a better understanding of the performance for different values of $a$, in Fig. 6 we show the convergence of the error for variations of the pycnocline sharpness. For moderate time steps and small values of $a$, the $M 1$, M2, PC2 and S15 scheme can treat the pycnocline as a barrier (Fig. 6 a). However, for values of $a \geq 7$, all schemes fail this test. Only by decreasing the time step to $10^{-6}$, the $M 1, M 2, P C 2$ and $S 15$ schemes show a scaling of the error over the whole range of variations of $a$ (Fig. 6 b). Clearly, the $S 15$ scheme shows the best performance. Again the $E 1$ scheme and $P C 1$ scheme do not treat the pycnocline correctly for all values of $a$.
Fig. 4 Dispersion of a particle cloud initially located at $z=0.75$ for two different schemes and two sets of $a$. Colour-coded is the particle concentration computed by box counting, for a $E 1$ scheme with $a=1, \mathbf{b} E 1$ with $a=4$, c $M 2$ with $a=1$ and d $M 2$ with $a=4$. The time step is $\Delta t=10^{-6}$
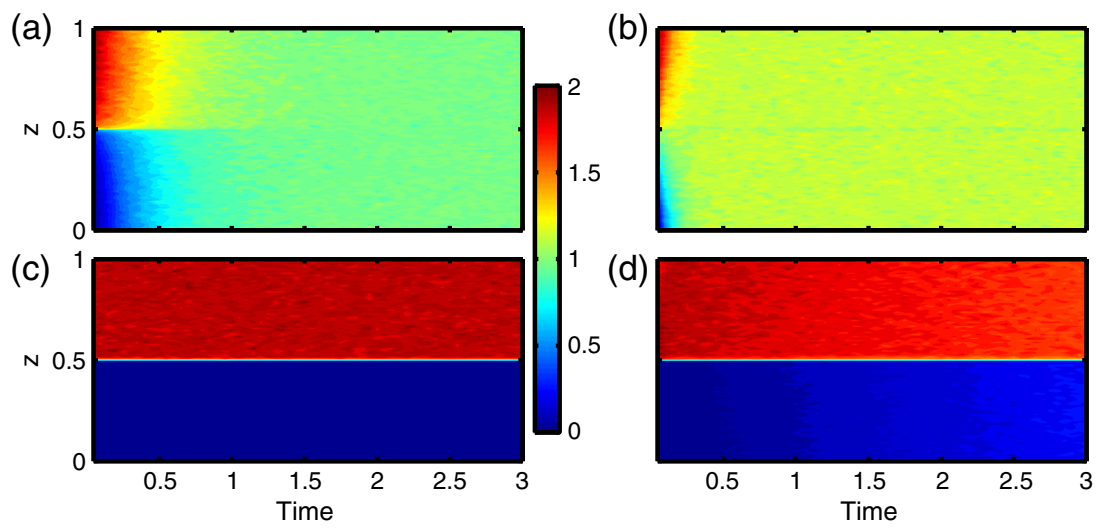
Fig. 5 Variation of the error $\varepsilon$ for the different numerical schemes and for a fixed parameter $a$ with: $\mathbf{a} a=1$ and b $a=3$. On the $x$ axis, we show the time step $\Delta t$ and on the $y$ axis the error $\varepsilon$
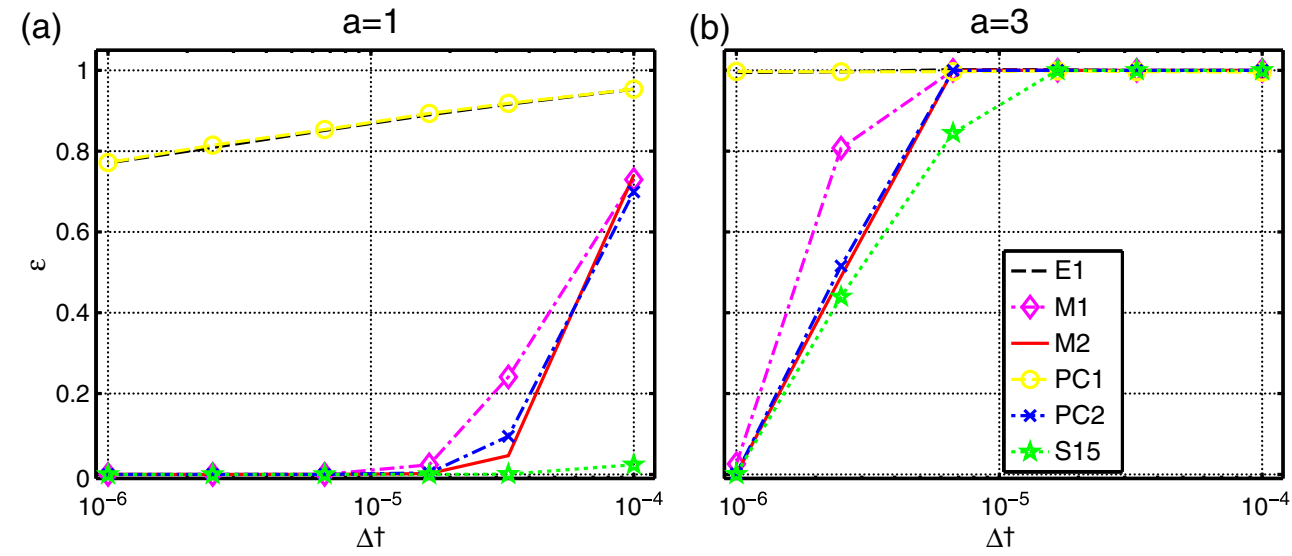

\subsubsection{Spurious background diffusivity}

There is an important question that has yet to be answered: Can we quantify the particle leakage introduced by the numerical schemes in terms of a physical quantity (rather than in the number of particles)? To answer the question, one has to recall Fig. 4 d. One can see that at the beginning, there exists a well-defined sharp interface at the pycnocline with a concentration of two above the pycnocline and zero below. As time progresses, there is a constant leakage of particles into the lower half of the water column. As this is a pure diffusion problem, it can be written as:

$\partial_{t} C=\partial_{z}\left(K \partial_{z} C\right)$.

We do not focus on the particle dynamics itself, but rather on the change in concentration such that there is a flux of concentration (particles) from top to down. If the pycnocline is a "true" barrier, the leakage is zero. However, since this is not the case, one can imagine that there exists a spurious background diffusivity that leads to the levelling of the concentration. Assuming that the spurious background diffusivity $K_{S}$ is constant, Eq. 20 can be written as:

$\partial_{t} C=K_{S} \partial_{z z} C$.

As we are seeking to estimate $K_{S}$ rearranging the terms leads to,

$K_{S}=\frac{\partial_{t} C}{\partial_{z z} C}$.

To finally estimate $K_{S}$, one can use any standard textbook method to solve a diffusion equation with constant coefficient, for instance a forward in time and central difference scheme. One has to note that we have to solve the inverse problem and do a least square fitting for the estimated diffusion constant.

In Fig. 7, the scaling of $K_{S}$ for variation of the time step is shown (keeping $a=1$ ). The $M 1, M 2, P C 2$ and $S 15$ schemes show a rapid decrease of $K_{S}$ by decreasing $\Delta t$ and finally reach the point where $K_{S}$ is equal zero. The $E 1$ and $P C 1$ schemes only show a very slow convergence of $K_{S}$. This still holds if we chose a time step that is two orders of magnitude below the
Fig. 6 Variation of the error $\varepsilon$ for the different numerical schemes and a fixed time step, with a $\Delta t=7 \times 10^{-6}$ and $\mathbf{b} \Delta t=10^{-6}$. On the $x$ axis, we show the pycnocline sharpness parameter $a$ and on the $y$ axis the error $\varepsilon$

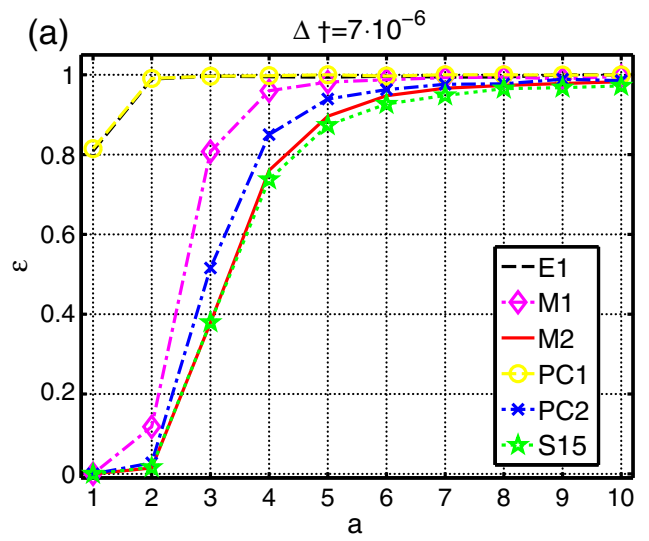

(b)

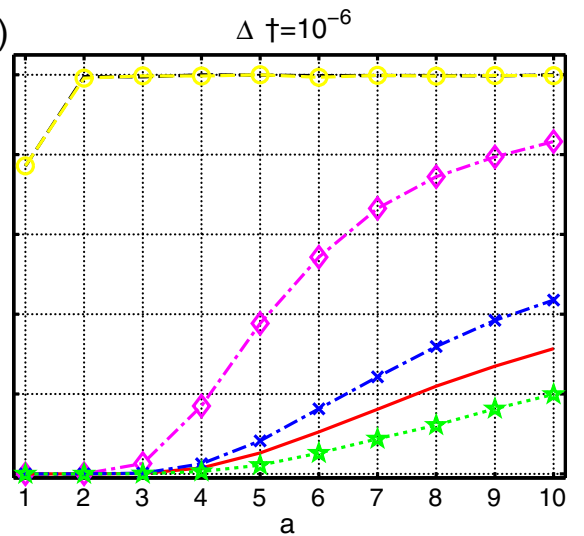




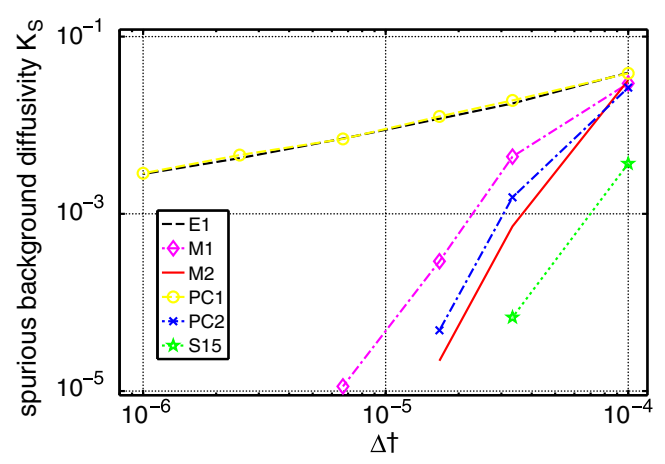

Fig. 7 Scaling of the background diffusivity $K_{S}$ for variations of the time step $\Delta t$ with fixed $a=1$. On the $y$ axis the error $\varepsilon$ is shown

time step restriction proposed by Visser (1997). The minimum background diffusivity for both schemes is approximately $5 \times 10^{-2}$. Since this is a dimensionless value, we have to convert it back to real physical units. Assume the diffusivity profile given in Fig. 1 with a depth averaged diffusivity of approximately $10^{-4} \mathrm{~m}^{2} / \mathrm{s}$. This gives a spurious background diffusivity for the Euler scheme of $5 \times 10^{-6} \mathrm{~m}^{2} / \mathrm{s}$. Thus, the E1 and $P C 1$ scheme would "ignore" diffusivities below that (which can reach down to $10^{-7} \mathrm{~m}^{2} / \mathrm{s}$ ). The $E 1$ scheme will overestimate the vertical diffusive fluxes by two orders of magnitude.

Figure 7 further indicates that particle-tracking schemes can show numerical diffusion, which is known from discretised PDEs in ocean models (Burchard and Rennau 2008; Vitousek and Fringer 2011). Nonetheless, for sufficiently small time steps and a proper numerical particle-tracking scheme, this artificial diffusivity can completely be removed.

\subsection{The residence time}

In the second test case, we compute the residence time of particles in the water column. In Fig. 8a, the scaling of the error is shown for $a=1$ and $P e=3$. All schemes converge to the analytic solution according to their proposed accuracy. It is important to note that not only the scaling is important, also the absolute error has to be considered. For instance, the $M 1$ scheme with the largest time step gives a similar error as the E1 scheme with the smallest time step and is thus much more efficient. The E1 scheme and PCI scheme show an identical performance. The same is valid for the second order schemes. Increasing the Peclet number (Fig. 8c) does not yield any new results. Only the absolute error for all schemes is slightly reduced, as with increased Peclet number, the particle movement close to the pycnocline is mainly controlled by the sinking speed (Fig. 3).

As discussed above, the residence time is an integral measure; thus, this test is a weak convergence problem. As the true particle path is not important, only the time evolution of a distribution is, all schemes show the proposed convergence behaviour. The $E 1$ scheme shows a similar performance compared to the $M 1$ scheme.

The error of the particle-tracking schemes does not show a strong dependence on the pycnocline sharpness (Fig. 8b). For the $E 1$ scheme and $P C 1$ scheme, $\varepsilon$ is even independent of the pycnocline sharpness.
Fig. 8 Results of the residence time test for: a $a=1$ and $\mathrm{Pe}=3$, b $\Delta t=10^{-5}$ and $\mathrm{Pe}=3$, c $a=1$ and $\mathrm{Pe}=12$. In $\mathbf{d}$, the efficiency (runtime vs. error) is shown for $a=1$ and $\mathrm{Pe}=3$. (a)

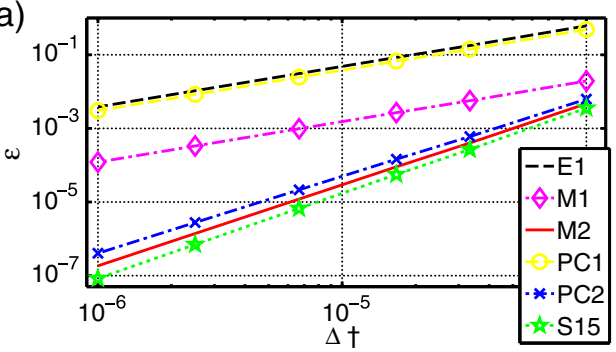

(c)

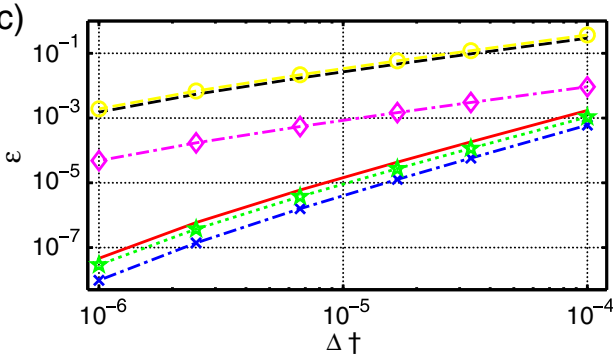

(b)

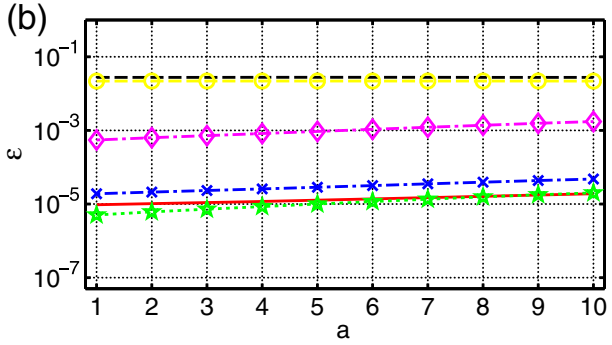

(d)

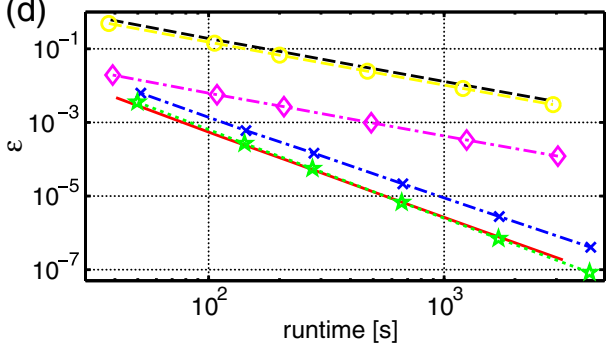


The efficiency of the different schemes, as indicated in Fig. 8 d, clearly shows the excellent performance of the second-order schemes. They can be regarded as highly efficient. It is further visible that the $M 1$ scheme has an error that is one order of magnitude lower than for the $E 1$ scheme and has a much higher efficiency.

\section{Why the Euler scheme fails}

Finally, we try to answer the question as to why the Euler scheme fails. At first, one has to recall the numerical schemes used. In Eq. 21, we have rewritten the $E 1$ and $M 1$ scheme in a more convenient way to have a better comparison between both:

$Z_{n+1}=Z_{n}+\partial_{z} K \Delta t+\sqrt{2 K} \Delta W: E 1$ scheme

$Z_{n+1}=Z_{n}+\partial_{z} K \frac{1}{2}\left(\Delta W^{2}+\Delta t\right)+\sqrt{2 K} \Delta W: M 1$ scheme.

The difference between the $E 1$ scheme and $M 1$ scheme is rather subtle. The correction for the $M 1$ scheme is only applied to the gradient term $\partial_{z} K$. Since

$(\Delta W)^{2}=O(\Delta t)$,

and $(\Delta W)^{2}$ is a random variable with mean and standard deviation $\Delta t$, the $M 1$ scheme tends to the E1 scheme for average size noise increments. Only for large or small noise increments the gradient corrections gets different weight. Consider for instance a particle close to the pycnocline and a noise increment $\Delta W=-2 \sqrt{\Delta t}$. For the $E 1$ scheme, the particle can cross the pycnocline. For the $M 1$ scheme, however, the large increment is balanced by an additional "repelling" force which is proportional to $3 / 2 \partial_{z} K \Delta t$. That might prevent a crossing. This indicates that noise increments larger than $\sqrt{\Delta t}$ lead to the poor performance of the E1 scheme. To test our hypothesis, we will modify the noise term $\Delta W$. The noise increment is usually a Gaussian variable (Eq. 3). As discussed above, we are seeking a solution that should converge in the weak sense; therefore, only the statistics of the noise increment $\Delta W$ need to be approximated in the weak sense. Thus, only its first two moments have to match. This means either any two-point random variable with

$P\left(\Delta W_{T}= \pm \sqrt{\Delta t}\right)=\frac{1}{2}$,

or a uniform distributed random variable as,

$W_{U}(t)=\left(U(t)-\frac{1}{2}\right) 2 \sqrt{3 \Delta t}$,

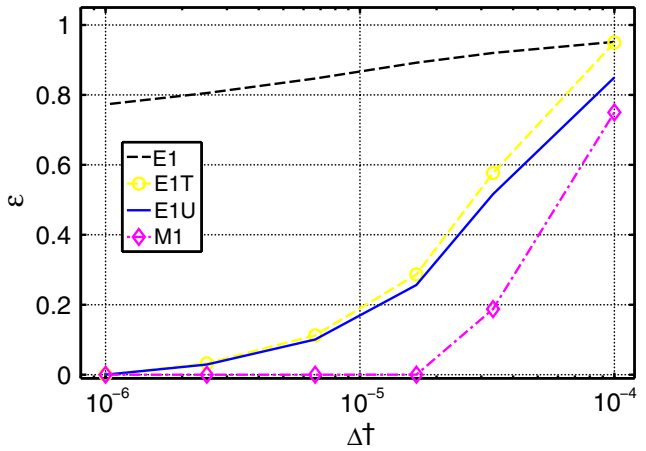

Fig. 9 Variation of the error $\varepsilon$ for different noise increments of the $E 1$ scheme with $a=1$. On the $y$ axis the error $\varepsilon$ is shown. As reference, the $M 1$ scheme is included

with $U(t)$ an uniform random number in the interval $[0,1]$ could be used. Both random variables fulfil the following necessary conditions:

$\langle W(t)\rangle=0 ; \operatorname{Std}(W(t)-W(s))=\sqrt{|t-s|}$.

Either $W_{T}(t)$ or $W_{U}(t)$ can be considered as a weak approximation of the original Wiener increment as defined in Eq. 3. A more profound discussion on approximating the moments of a distribution can be found in Kloeden and Platen (1999). However, it is important to note that by using either $W_{T}(t)$ or $W_{U}(t)$, the $E 1$ scheme is still only of first-order accuracy in the weak sense (Eq. 5), whereas the convergence in the strong sense is not defined any longer.

To test our hypothesis, we repeat the diffusive pycnocline crossing experiment as discussed in Section 3.2. The numerical schemes under considerations are the E1 scheme, the Euler scheme with a two-point random variable $\Delta W_{T}$ is the E1T scheme and the Euler scheme with a uniform random variable $\Delta W_{U}$ is the $E 1 U$ scheme. As an additional benchmark solution, we use the $M 1$ scheme.

Figure 9 clearly indicates that the bounded noise increments (Eq. 22 or 23) improve the performance of the $E 1$ scheme. Especially for small time steps, the E1T and E1U schemes converge to the "true" solution so that the pycnocline is a quasi-impermeable barrier to diffusion. However, the $M 1$ scheme can clearly outperform the modified $E 1$ schemes; thus, it is still beneficial to use an improved particle-tracking scheme and not a "fixed" one.

\section{Conclusion}

In this paper, we described a new test case for particletracking schemes. The motivation to construct this benchmark was to investigate how different numerical 
schemes handle variations in the diffusivity by several orders of magnitude and sharp interfaces as represented by a well-defined pycnocline. Moreover, the proposed test can be used to assess the weak and strong convergence properties of various numerical schemes.

The findings of this paper can be summarised in one sentence: Do not use the Euler scheme for particle tracking in highly baroclinic simulations! In particular, the diffusive pycnocline crossing test revealed that the E1 scheme or PC1 scheme is of no use, since they do not treat the pycnocline as an impermeable boundary. Even lowering the time step did not solve the problem. The analysis showed that the poor performance of both schemes is related to a high spurious background diffusivity. This artificial diffusivity limits the range of variations in the "real" diffusivity. The poor performance of the E1 scheme or PCI scheme is not correlated with the theoretical convergence of the error, which is for both schemes of first order. The Milstein scheme, which is also of first order, showed much better ratings, a higher efficiency and accessorily an absolute error that is approximately one order of magnitude lower compared to the E1 scheme. This gain is caused by an additional correction term, which accounts for the size of the noise increment. A second reason for the good performance of the Milstein scheme is that the strong error convergence is better than for the E1 scheme. Although particle tracking is mostly used to describe the time evolution of distributions rather than individual tracks, close to boundaries (and the pycnocline is such a boundary), the individual particle path is important and hence the performance in the strong sense. Similar suggestions were made by Stijnen et al. (2006) or Gräwe and Wolff (2010).

The failure of the Euler scheme was only apparent in the diffusive pycnocline crossing test. For the residence time, the E1 scheme or PC1 scheme showed a proper scaling, although the absolute error was at least one order of magnitude higher than the other schemes. If the sinking velocity is small compared to the typical diffusion length, the usage of low order particletracking schemes is highly questionable. In that limit, it is highly recommended to turn to higher order schemes.

A possible solution to improve the Euler scheme is to use bounded noise increments (Eq. 22 or 23). The drawback of the modification is that the Euler scheme has only a well-defined convergence in the weak sense. The strong convergence is no longer defined. By using a large numbers or particles, the distribution of particles is well sampled. Especially the uniform noise increment (Eq. 23) is commonly used. The reason is that uniform random numbers are faster to generate than Gaussian ones. However, as common applications deal with sim- ulating individual tracks of drifter, species or larvae, the tracking scheme is applied in the strong sense (Eq. 5), where the convergence of the modified Euler scheme is not defined.

The results of the pycnocline crossing test suggest that existing particle-tracking schemes should be updated to at least the Milstein scheme. This would only require minor modifications in the commonly used Euler scheme but will be rewarded by a much higher accuracy. Additionally, the efficiency is much higher, meaning that the overhead due to a more complex numerical scheme is cancelled out by the much higher accuracy and therefore lower computational cost.

Acknowledgements Eric Deleersnijder was a research associate with the Belgian Fund for Scientific Research (F.R.S.FNRS). His contribution to the present study was achieved in the framework of the Interuniversity Attraction Pole TIMOTHY, which is funded by BELSPO under the contract IAP6.13, and the ARC 10/15-028 (Communauté Française de Belgique). Ulf Gräwe was funded by the Bundesministerium für Bildung, Wissenschaft, Forschung und Technologie of Germany (BMBF) through grant number 01LR0807B.

\section{References}

Arnold L (1974) Stochastic differential equations: theory and applications. Wiley, London

Beron-Vera FJ, Olascoaga MJ (2009) An assessment of the importance of chaotic stirring and turbulent mixing on the West Florida Shelf. J Phys Oceanogr 39(7):1743-1755

Blanke B, Raynaud S (1997) Kinematics of the Pacific equatorial undercurrent: an Eulerian and Lagrangian approach from GCM results. J Phys Oceanogr 27(6):1038-1053

Blumberg A, Dunning D, Li H, Heimbuch D, Rockwell Geyer W (2004) Use of a particle-tracking model for predicting entrainment at power plants on the Hudson River. Estuar Coast 27:515-526

Brickman D, Smith PC (2001) Lagrangian stochastic modeling in coastal oceanography. J Atmos Ocean Technol 19(1):83-99

Brochier T, Lett C, Tam J, Fréon P, Colas F, Ayón P (2008) An individual-based model study of anchovy early life history in the northern Humboldt Current system. Prog Oceanogr 79(2-4):313-325

Burchard H, Rennau H (2008) Comparative quantification of physically and numerically induced mixing in ocean models. Ocean Model 20(3):293-311

Callies U, Plüß A, Kappenberg J, Kapitza H (2011) Particle tracking in the vicinity of Helgoland, North Sea: a model comparison. Ocean Dyn 1-19

Christensen A, Daewel U, Jensen H, Mosegaard H, St John M, Schrum C (2007) Hydrodynamic backtracking of fish larvae by individual-based modelling. Mar Ecol Prog Ser 347:221232

de Brauwere A, Deleersnijder E (2010) Assessing the parameterisation of the settling flux in a depth-integrated model of the fate of decaying and sinking particles, with application to fecal bacteria in the Scheldt Estuary. Environ Fluid Mech 10:157-175 
Deleersnijder E, Beckers JM, Delhez EJM (2006a) On the behaviour of the residence time at bottom of the mixed layer. Environ Fluid Mech 6:541-547

Deleersnijder E, Beckers JM, Delhez EJM (2006b) The residence time of settling in the surface mixed layer. Environ Fluid Mech 6:25-42

D’Ovidio F, Fernández V, Hernández-García E, López C (2004) Mixing structures in the Mediterranean Sea from finite-size Lyapunov exponents. Geophys Res Lett 31:L17203

Elliott AJ, Dale AC, Proctor R (1992) Modelling the movement of pollutants in the UK shelf seas. Mar Pollut Bull 24(12):614-619

Gräwe U (2011) Implementation of high-order particle-tracking schemes in a water column model. Ocean Model 36(1-2):8089

Gräwe U, Wolff JO (2010) Suspended particulate matter dynamics in a particle framework. Environ Fluid Mech 10(1):2139

Huret M, Runge JA, Chen C, Cowles G, Xu Q, Pringle JM (2007) Dispersal modeling of fish early life stages: sensitivity with application to Atlantic cod in the western Gulf of Maine. Mar Ecol Prog Ser 347:261-274

Iskandarani M, Levin J, Choi BJ, Haidvogel DB (2005) Comparison of advection schemes for high-order h-p finite element and finite volume methods. Ocean Model 10(1-2):233252

Kloeden P, Platen E (1999) Numerical solution of stochastic differential equations (stochastic modelling and applied probability), 3rd edn. Springer, Berlin

Krestenitis YN, Kombiadou KD, Savvidis YG (2007) Modelling the cohesive sediment transport in the marine environment: the case of Thermaikos Gulf. Ocean Sci 3(1):91104

LaBolle EM, Quastel J, Fogg GE, Gravner J (2000) Diffusion processes in composite porous media and their numerical integration by random walks: generalized stochastic differential equations with discontinuous coefficients. Water Resour Res 36(3):651-662

Lalescu C, Teaca B, Carati D (2010) Implementation of high order spline interpolations for tracking test particles in discretized fields. J Comput Phys 229(17):5862-5869

Lane A, Prandle D (2006) Random-walk particle modelling for estimating bathymetric evolution of an estuary. Estuar Coast Shelf Sci 68(1-2):175-187

Mariano A, Kourafalou V, Srinivasan A, Kang H, Halliwell G, Ryan E, Roffer M (2011) On the modeling of the 2010 Gulf of Mexico oil spill. Dyn Atmos Ocean 52(1-2):322340

Marsaglia G (2003) Xorshift RNGs. J Stat Software 8(14):1-6

Marsaglia G, Tsang WW (2000) The ziggurat method for generating random variables. J Stat Software 5(8):1-7

Maruyama G (1955) Continuous Markov processes and stochastic equations. Rend Circ Mat Palermo 4:48-90

Milstein GN (1974) Approximate integration of stochastic differential equations. Theory Probab Appl 19:557-562
Milstein GN (1979) A method of second-order accuracy integration of stochastic differential equations. Theory Probab Appl 23(2):396-401

North E, Hood R, Chao SY, Sanford L (2006) Using a random displacement model to simulate turbulent particle motion in a baroclinic frontal zone: a new implementation scheme and model performance tests. J Mar Syst 60(3-4):365-380

Ohlmann JC, Mitarai S (2010) Lagrangian assessment of simulated surface current dispersion in the coastal ocean. Geophys Res Lett 37:L17602

Penland C (2003) A stochastic approach to nonlinear dynamics: a review. Bull Am Meteorol Soc 84(7):43-52

Pietrzak J (1998) The use of TVD limiters for forward-in-time upstream-biased advection schemes in ocean modeling. Mon Weather Rev 126:812-830

Prather MJ (1986) Numerical advection by conservation of second-order moments. J Geophys Res 91:197-221

Proehl JA, Lynch DR, McGillicuddy DJ, Ledwell JR (2005) Modeling turbulent dispersion on the North Flank of Georges Bank using Lagrangian particle methods. Cont Shelf Res 25(7-8):875-900

Shah SHAM, Heemink AW, Deleersnijder E (2011) Assessing Lagrangian schemes for simulating diffusion on non-flat isopycnal surfaces. Ocean Model 39(3-4):351-361

Silverman BW (1986) Density estimation for statistics and data analysis (Chapman \& Hall/CRC monographs on statistics \& applied probability), 1st edn. Chapman and Hall/CRC, Boca Raton

Soomere T, Andrejev O, Myrberg K, Sokolov A (2011) The use of Lagrangian trajectories for the identification of the environmentally safe fairways. Mar Pollut Bull 62(7):1410 1420

Spivakovskaya D, Heemink A, Deleersnijder E (2007a) Lagrangian modelling of multi-dimensional advectiondiffusion with space-varying diffusivities: theory and idealized test cases. Ocean Dyn 57(3):189-203

Spivakovskaya D, Heemink AW, Deleersnijder E (2007b) The backward Îto method for the Lagrangian simulation of transport processes with large space variations of the diffusivity. Ocean Sci 3(4):525-535

Stijnen JW, Heemink A, Lin HX (2006) An efficient 3D particle transport model for use in stratified flow. Int J Numer Methods Fluids 51(3):331-350

Thomson DJ (1987) Criteria for the selection of stochastic models of particle trajectories in turbulent flows. J Fluid Mech 180:529-556

van der Lee EM, Umlauf L (2011) Internal-wave mixing in the Baltic Sea: Near-inertial waves in the absence of tides. J Geophys Res 116(C10016)

Visser AW (1997) Using random walk models to simulate the vertical distribution of particles in a turbulent water column. Mar Ecol Prog Ser 158:275-281

Vitousek S, Fringer OB (2011) Physical vs. numerical dispersion in nonhydrostatic ocean modeling. Ocean Model 40(1):7286 